\title{
Chemical Determinants of the Rat Electro-Olfactogram
}

\author{
John W. Scott, Tracy Brierley, and Frederick H. Schmidt \\ Department of Cell Biology, Emory University School of Medicine, Atlanta, Georgia 30322-3030
}

The chemical properties that determine the distribution of the electro-olfactogram were studied after exposure of a large area of the rat olfactory epithelium. Multiple electrodes were placed along the rostral border of endoturbinate IV on the midline of the nasal cavity. This array of electrodes spanned a region containing the four receptor gene expression zones described for the rat. The response to a series of odorants containing only carbon, hydrogen, and oxygen was strongly related to electrode position. For most hydrocarbons, the responses were progressively larger toward the ventral epithelium. The only exceptions were aromatic hydrocarbons, which evoked nearly equal response sizes across the epithelium. Ketones and aldehydes evoked relatively larger dorsal responses than did hydrocarbons with similar structures. Aromatic ketones and aldehydes evoked systematically larger responses from the dorsal

The understanding of olfactory coding has been greatly changed by two recent sets of findings. The first was the finding that rabbit mitral/tufted cells of a particular part of the dorsomedial olfactory bulb are sensitive to aliphatic acids, aldehydes, and esters (Mori and Yoshihara, 1995). Individual cells in this part of the bulb also respond maximally to odorous compounds of a particular size. Recent behavioral data (Linster and Hasselmo, 1999) have demonstrated that generalization across a series of aliphatic aldehydes is predictable from those mitral/tufted cell responses. These experiments show promise of developing a series of dimensions that will ultimately describe the olfactory stimulus space.

The second important set of findings stemmed from the discovery of the olfactory receptor gene family (Buck and Axel, 1991), which led to the ability to localize the expression of these genes by in situ hybridization (Nef et al., 1992; Ressler et al., 1993, 1994; Vassar et al., 1993, 1994; Strotmann et al., 1994; Kubick et al., 1997) and other techniques (Mombaerts et al., 1996). These studies showed that receptor expression is localized in the epithelium of rats and mice. In addition, any receptor gene is associated with axons converging onto a small subset of glomeruli, often one medial glomerulus and one lateral glomerulus. This convergence pattern provides a potential explanation for the functional specificity of mitral cell recordings (Buonviso and Chaput, 1990; Mori and Yoshihara, 1995) and of metabolic markers of glomerular function (Guthrie et al., 1993; Johnson et al., 1998, 1999).

\footnotetext{
Received Jan. 27, 2000; revised April 4, 2000; accepted April 4, 2000.

This work was supported by the National Institute of Deafness and Other Communications Disorders Grant DC 00113. We thank Dr. M. Chaput for reading a previous draft of this manuscript and Dr. D. Goldsmith for suggestions.

Correspondence should be addressed to Dr. John W. Scott, Department of Cell Biology, 1648 Pierce Drive, Emory University School of Medicine, Atlanta, GA 30322-3030. E-mail: JohnS@cellbio.emory.edu.

Copyright (C) 2000 Society for Neuroscience $\quad 0270-6474 / 00 / 204721-11 \$ 15.00 / 0$
}

part of the epithelium. The response profiles for most odorants were well described by a linear fit to the electrode position along the dorsal-ventral position on the epithelium. However, a few bicyclic odorants and carboxylic acids evoked significantly nonlinear profiles. It is concluded that there is a systematic distribution of odorant sensitivity across this part of the epithelium and that this sensitivity is related to general chemical properties. Other evidence suggests that these properties extend to other parts of the epithelium. This spatial sensitivity of the epithelium to odorants probably contributes to olfactory coding in parallel with the convergence of axons from olfactory sensory neurons expressing the same receptor type.

Key words: electro-olfactogram; odorant; rat; hydrocarbon; ketone; olfactory epithelium

Our laboratory has attempted to integrate some of these findings with electro-olfactogram (EOG) recordings from the olfactory epithelium. The EOG is a slow potential recorded extracellularly from a population of olfactory sensory neurons. We found a general correspondence between the gene expression zones described for neonatal rat and mouse olfactory epithelium (Ressler et al., 1993; Vassar et al., 1993) and the distributions of sensitivities of response on the exposed adult olfactory epithelium to a set of terpene odors (Scott et al., 1997; Scott and Brierley, 1999). We investigated a wider series of odors in an intact animal (Scott et al., 1996), but this preparation did not allow direct comparison with the receptor gene expression zones. In addition, the intact nasal cavity is subject to influences from differential sorption of odorants that alters the concentration in different parts of the epithelium (Ezeh et al., 1995; Kent et al., 1996) (P. E. Scott-Johnson, D. Blakley, and J. W. Scott, unpublished observations). In the present series, we have investigated the distribution of responses to a series of aliphatic, terpene, and aromatic compounds and related odorants. The series were chosen to investigate the structural relationships with the terpene odors that we had tested previously. That is, the effects of different chemical structures such as open chains versus cyclic structures and of different functional groups were explored where we could find appropriate commercially available stimuli. These results show systematic differences between saturated hydrocarbon odorants, which do not have polar bonds, and structurally similar compounds containing oxygen, which adds an asymmetric charged group to the molecule. These predictable relationships in the responses may be important in sensory function.

\section{MATERIALS AND METHODS}

Surgical preparation. Male Sprague Dawley rats (375-525 gm) were killed with an overdose of Nembutal $(20 \mathrm{mg} / \mathrm{kg})$ and placed in a stereotaxic frame. The medial surface of the olfactory epithelium overlying the 
endoturbinate bones on the left side was exposed as in our previous papers (Scott et al., 1997; Scott and Brierley, 1999). The endoturbinate bones are the protrusions of the ethmoid bones that extend close to the midline of the nasal cavity. The entire region from endoturbinate II to endoturbinate IV was exposed from the dorsal margin at the cribriform plate to the ventral margin at the respiratory epithelium. The room temperature was kept cool $\left(\sim 17^{\circ} \mathrm{C}\right)$ to prolong the usefulness of the preparation. A room humidifier and plastic sheeting were used to maintain high humidity near the preparation. A constant flow of humidified air (see below) was immediately directed over the epithelium.

Recording procedure. Simultaneous recordings were made with four or eight glass micropipettes filled with agar made up in Ringer's solution. These micropipettes were broken to obtain resistances $<5 \mathrm{M} \Omega$. The leads from these electrodes were connected to four-channel AC-coupled amplifiers (low-frequency cutoff of $0.1 \mathrm{~Hz}$ and high-frequency cutoff of 30 $\mathrm{Hz}$ ). The electrodes were placed with independent manipulators, but we spaced them as equally as possible along the rostral borders of endoturbinate IV. Placement of the electrodes was monitored by listening to an audio monitor output from the amplifiers to fix the electrodes at the point of first contact with the tissue. This should place the electrode at the mucosal surface and give the maximal response amplitude. It is likely, however, that some electrodes advanced into the tissue slightly as others were being set. The electrodes usually remained in place during a complete recording session, but on rare occasions two sets of placements were used during a single experiment. Electrodes were reset in the same position if vibration or slight tissue drying resulted in loss of contact. An indifferent electrode (a silver chloride electrode connected by an agar bridge to a saline-soaked cotton pad) was placed on the frontal bone.

Odor stimulation and computer control. Odorants were injected into a humidified, clean air stream flowing at $1000 \mathrm{ml} / \mathrm{min}$. This air stream, in turn, flowed into a chamber with two large ports for clean air input and vacuum. Before each stimulus, the odor was turned on for $20 \mathrm{sec}$ to allow the buildup of the odor in the system. During this buildup period, the vacuum port was opened to wash the odor from the stimulus port and prevent stimulation. The vacuum line flowed in excess of $1000 \mathrm{ml} / \mathrm{min}$. The actual rate was adjusted to give no EOG response at the onset of buildup or no upward drift at the presentation of blanks. Stimulation was applied to the epithelium by the closing of the vacuum port. This procedure is similar to that described by Kauer and Shepherd (1975) or by Mackay-Sim and Kesteven (1994). Although it is likely that there were some transients of airflow during these valve operations and some differences between the total flow in the buildup and stimulation periods, the blanks usually produced responses of $<0.4 \mathrm{mV}$. If the blanks became larger, the system was flushed with clean air to reduce contamination. The odor port was placed $1 \mathrm{~cm}$ from the epithelium and centered over the recording area. The port approached the epithelium from below the bar of the stereotaxic frame at an angle of $\sim 30^{\circ}$. In a previous paper (Scott et al., 1997), we could detect no influence of the tube angle on the differential distribution of odor responses.

Odor stimuli were generated with an air dilution olfactometer, and concentration was expressed as a proportion of air saturated with odorant. A syringe pump forced air through the head space in glass bottles in which $2-5 \mathrm{ml}$ of the odorant was used to cover the bottom. The odorant sample bottles were connected by Teflon tubes to ports in the glass tube next to the epithelium. A BASIC language program controlled the pump rate and set valves to inject each odor. This program used a set of standard files to determine the odor sequence and concentration. Stimuli were presented in sets of seven, with each set preceded by a standard isoamyl acetate stimulus at a dilution of $10^{-1}$ and followed by a blank. In addition to the standard isoamyl acetate stimulus, each set began with the three terpene odorants D-carvone, D-limonene, and 1,8-cineole. We have used these stimuli extensively before, and their use ensured that consistent responses were obtained. Overall, results with the eight electrodes were obtained from 98 animals for these three odorants, with smaller numbers of animals for the other test odorants. Odorants were presented in a descending series of dilutions from $1 \times 10^{-1}$ to $1 \times 10^{-3} ; 1.5 \mathrm{~min}$ elapsed after each stimulus presentation. The recording session usually lasted 3-5 hr. We sometimes terminated an experiment earlier if the responses began to deteriorate badly on three or more electrodes. We were often able to repeat the concentration series several times or to substitute a different set of odorants. We always tested the terpene odorants at the end of the session to be sure that the response to these had remained stable. Because of this arrangement, we have much more extensive data for the three terpene odorants than for most other stimuli.

Data analysis. The amplifier outputs were fed through an analog-to- digital converter (digitized at a rate of $26 \mathrm{~Hz}$ ) to a computer that plotted the traces and computed the peak negative voltage relative to the baseline just before the stimulus. This peak voltage was printed for each plot and was also stored in a computer file for later statistical analysis. The plots were inspected for quality control during data collection and before analysis. Data were not used in the analysis if the standardized blank response was $>1 \mathrm{mV}$ or if the average response to the isoamyl acetate standard was $<4 \mathrm{mV}$. (Large blank responses were observed only when the response to the isoamyl acetate standard was very large.) If a preparation was excessively noisy or, in rare cases, it failed to show the response patterns normally evoked by the three terpene stimuli, the experiment was terminated.

Responses to all stimuli were standardized to the isoamyl acetate stimulus. This had two purposes. It helped minimize differences between recordings that might result from slight damage or drying at one site. It also allowed us to adjust for the gradual rundown of the response over the period of recording. The response to each odor was divided by the immediately previous response to the standard stimulus at that electrode and was multiplied by the best estimate of the optimal isoamyl acetate response. This estimate was established by averaging the initial response to the standard concentration of this stimulus $\left(1 \times 10^{-1}\right)$ over 10 preparations with four electrode recordings. Although there is considerable variation in the size of responses to the standard odor, the average of those responses changes little across the dorsal-to-ventral extent of endoturbinate IV (Scott and Brierley, 1999). The frequent presentation of the standard stimulus allowed us to correct for changes over time at each electrode independently. A second normalization was applied within each recording session to remove variation that may have come from slight leaks in the system or systematic differences between animals. In this normalization, the response at each electrode to each odor was divided by the average response to that odor for the particular session. This normalized response was then multiplied by the average response across all sessions for that odor and concentration.

For the statistical comparison of response variation across position and across recording sessions, we used stepwise multiple regression instead of ANOVA. A photograph of each epithelium was made with the electrodes in place, and the positions of the electrode tips were marked on this photograph. Photocopy enlargements were made of the marked photographs, and a standard transparent overlay with rectangular coordinates was used to record the positions of the tips. We used regression analysis for these data because it allowed comparison of odorants that were not recorded in the same preparation.

Response profiles (standardized EOG amplitudes obtained for given concentrations and electrode positions) were plotted for each odorant stimulus. In the simplest cases, the response profiles could be fit by a simple linear equation:

$$
Y=c_{1}+c_{2} \cdot X
$$

where $Y$ was the standardized response magnitude, $X$ was the position measured along the rostral border of endoturbinate IV (see Fig. $1 A$ ), and $c_{i}$ values were the intercept and slope of the line. For other profiles, a higher order polynomial regression formula was necessary for estimating the curve:

$$
Y=c_{1}+c_{2} \cdot X+c_{3} \cdot X^{2}+c_{4} \cdot X^{3},
$$

where $c_{i}$ values were the coefficients of the polynomial terms.

To test whether profiles had significant nonlinear components, we used an $F$ test based on the sum of squares in regressions with linear and nonlinear terms (Kleinbaum and Kupper, 1978):

$$
F=\frac{((\text { Sum of squares linear model })-}{(\text { Sum of squares total model } /(N-2))},
$$

where the degrees of freedom for the numerator are the difference in the number of parameters for the total model and the linear model. The degrees of freedom in the denominator have been reduced because of the normalization.

When we compared the linear slopes of two response profiles, we used the formula:

$$
Y=c_{1}+c_{2} \cdot X+D+c_{3} \cdot D \cdot X,
$$

where $D=0$ or $D=1$ is a dummy variable representing a comparison of two odors. The dummy variable is a standard regression technique that 
A

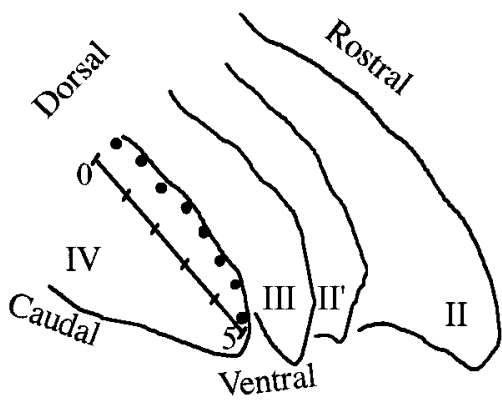

B

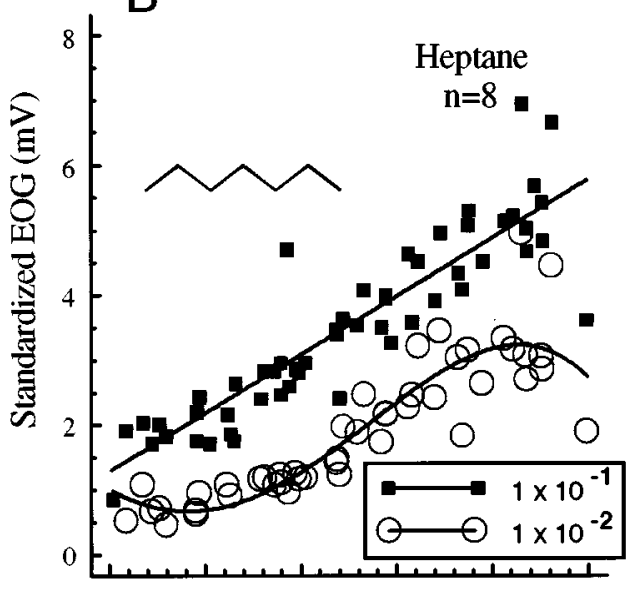

C

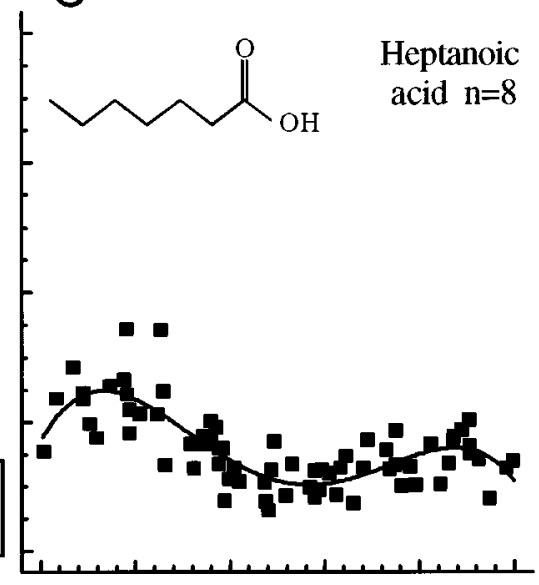

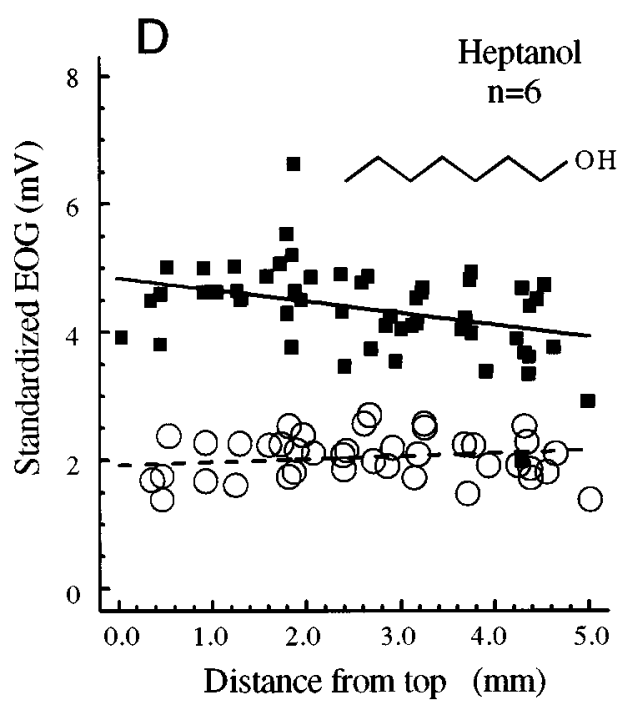
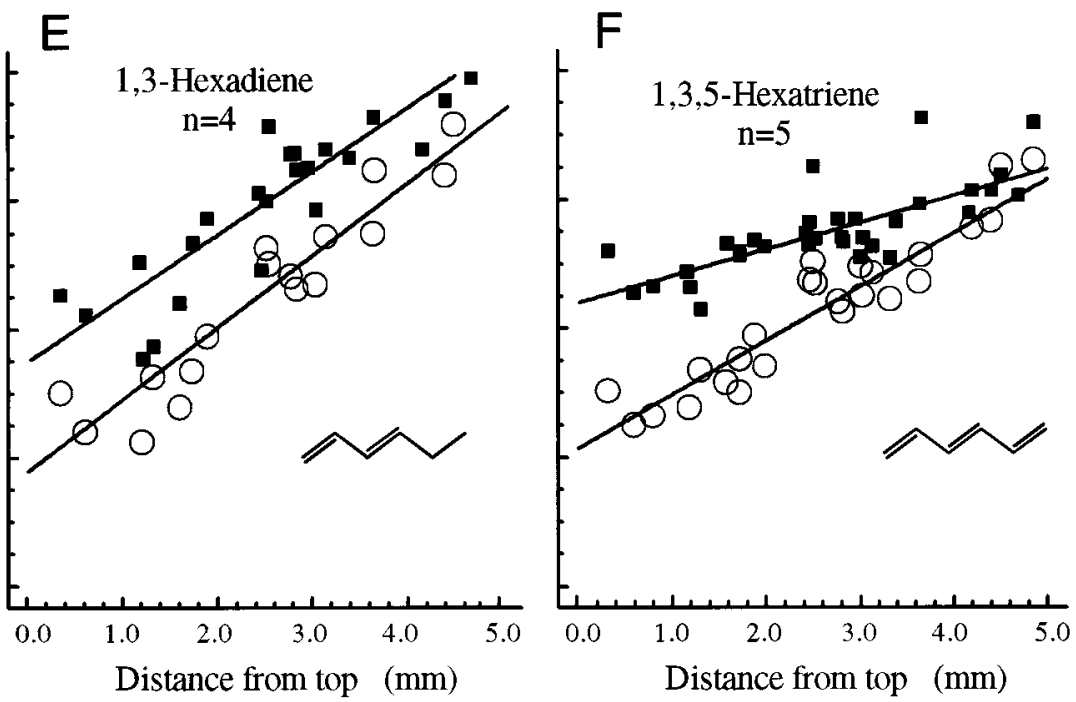

Figure 1. A, The positions of the eight electrodes ( filled circles) set up in each recording session. These electrodes were equally spaced along the rostral border of the midline of endoturbinate IV. $B-F$, The response profiles for a set of aliphatic odorants with six or seven carbons. The concentration key in $B$ applies to all the panels in this figure. We plotted responses as dilutions of $1 \times 10^{-1}$ and $1 \times 10^{-2}$ for most odorants in this figure and below (see Figs. 3-5). In some cases in which more extensive data existed, we also plotted the responses for $1 \times 10^{-3}$. For heptanoic acid $(C)$, we have plotted responses only at $3 \times 10^{-1}$ because they were very small at lower concentrations. All the plots of response profiles are presented on the same scale. The response profiles were fit with straight lines if the comparison of regression by first-order and third-order equations did not indicate a significant improvement in fit by the higher order equation. The numbers of rats are indicated for each panel. In $D$, the dashed line indicates that the regression is not significant.

allows comparison of multiple variables in a single analysis. In this case, it allowed us to test whether separating the points from two odors allowed a significantly better fit than fitting a single slope through all the points. The $F$ test for Equation 4 was:

$$
F=\frac{((\text { Sum of squares single-slope model })-}{(\text { Sum of squares two-slope model }))} .
$$

\section{RESULTS}

\section{Aliphatic odorants}

Figure $1 A$ shows the general placement of recording electrodes across the rostral border of endoturbinate IV, which was used in all the figures reported here. This view is similar to the wholemount display of olfactory expression zones shown by Vassar et al. (1993). When we refer to electrode numbers, the "number 1" electrode was the most dorsal, and the "number 8 " electrode was the most ventral. The scale shows the measurement in millimeters obtained from photographic overlays as described in Materials and Methods. Figure $1 B$ shows the distribution of standardized responses for the straight-chain hydrocarbon heptane. This distribution had a steep positive slope, in that the responses became larger as distance from the dorsal part of the epithelium increased. At the highest concentration $\left(1 \times 10^{-1}\right)$ the curve fit to the response profile was linear, whereas at $1 \times 10^{-2}$ there was a slight, but statistically significant, curvature evidenced by significant second- and third-order terms in the polynomial fit. The coefficients of determination for the linear and nonlinear equations were 0.78 and 0.82 , respectively, showing that the nonlinear terms account for less than a $4 \%$ difference in the variance. Figure 1, $C$ and $D$, shows distributions for heptanol and heptanoic acid (enanthylic acid). The addition of alcohol $(\mathrm{OH})$ or acid $(\mathrm{COOH})$ groups to the heptane molecule significantly changed the profiles. The slopes determined by linear regression of the heptanol and 

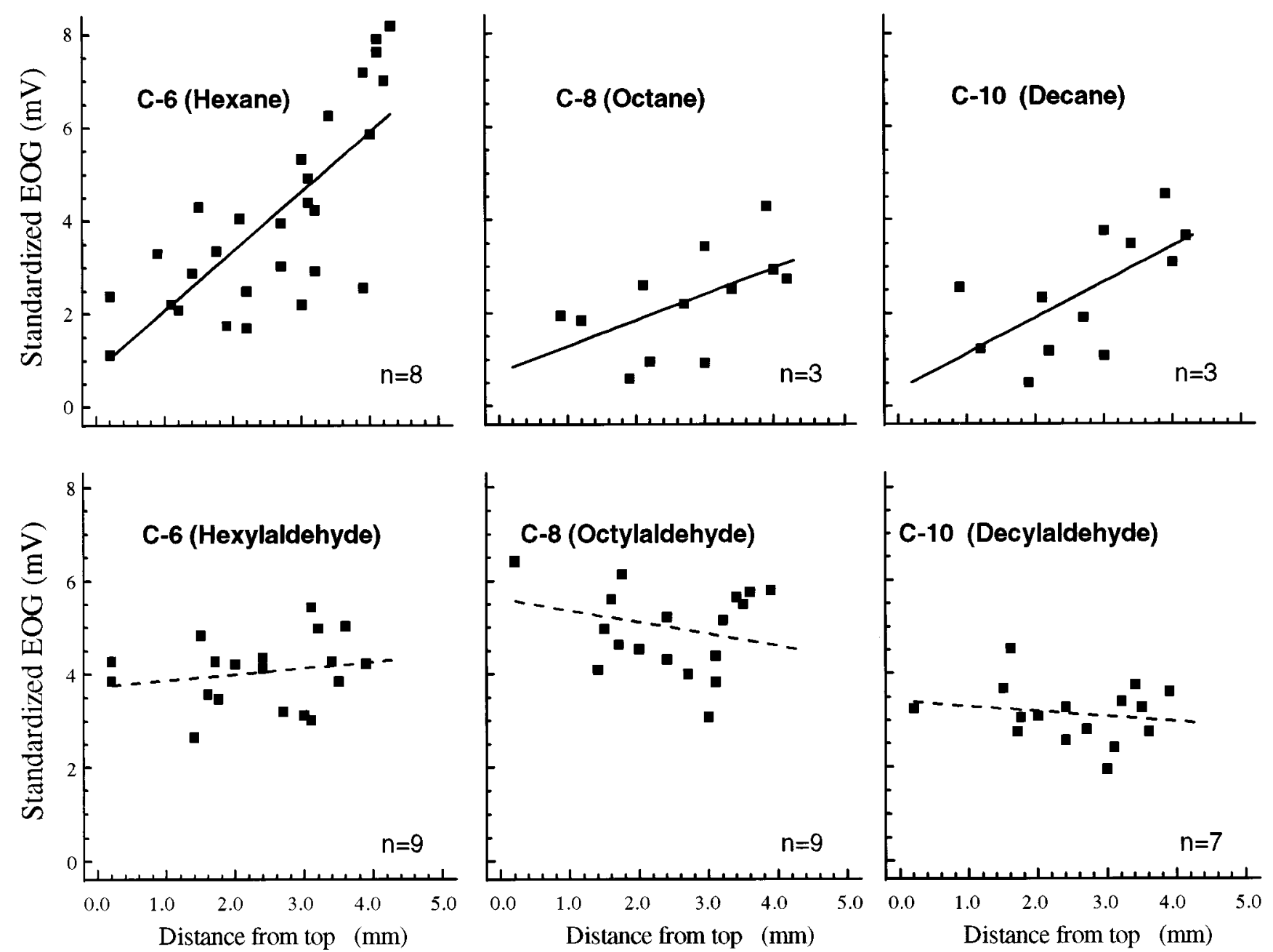

Figure 2. Aliphatic odorants tested with the four-electrode array. Top row, Three of the five straight-chain alkanes, identified by the number of carbons, are shown. Although both the hexane $(C-6)$ and heptane ( $C$-7; data not shown) profiles had slightly steeper slopes, none of the slopes in this panel were significantly different from each other. The numbers of animals are indicated in each panel. Bottom row, None of the aldehyde profile slopes were significantly different from each other, but each of the aldehyde profile slopes was significantly different $(p<0.05)$ from each of the alkane profile slopes. The dashed lines indicate that the regressions are not significant.

heptanoic acid profiles were significantly different from the heptane slope at any concentration. In addition, the stepwise regression analysis showed significance $(p<0.01)$ for the second- and third-order terms in the profile for heptanoic acid. We did not extensively study a series of carboxylic acids in this series because the responses were so small. However, tests of hexanoic acid at $1 \times 10^{-1}$ (data not shown) also showed a similar distribution that showed deviation from a linear profile with $p<0.05$.

The effect of adding double bonds to the unsaturated heptane molecule was small (Fig. $1 E, F$ ) and similar to that of the addition of oxygen-containing groups. The hexadiene and hexatriene odorants both produced stronger responses than heptane. In the case of hexatriene, it appears possible that the response reached a maximum, causing a flattening of the profile. Therefore, it was more appropriate to compare the heptane profile at $1 \times 10^{-1}$ with the hexadiene and hexatriene profiles at $1 \times 10^{-2}$. The slope for hexadiene was significantly $(p<0.01)$ more positive, but the slope for the hexatriene profile was not significantly different from that for heptane.

Because there have been several studies of the effect of chain length on response to aliphatic aldehydes or acids (Mori and
Yoshihara, 1995; Johnson et al., 1998, 1999; Krautwurst et al., 1998; Zhao et al., 1998; Linster and Hasselmo, 1999), we compared a series of aldehydes and alkanes in 11 rats using a fourelectrode array along the same extent of endoturbinate IV. These results are shown in Figure 2. Even though these recordings were conducted by a different person on a different setup, the profiles for hexane agree quite well with those collected with eight electrodes (data not shown). The profiles were fit by linear equations for simplicity of comparison. There were no significant differences in slope within either series across the dorsal-to-ventral extent of endoturbinate IV, but the slopes for each of the aldehyde profiles were different from each of the corresponding alkane profiles.

Figure 3 illustrates a series of cyclic compounds. In these cases, the profile for the saturated hydrocarbon cyclohexane had a steep positive slope with larger ventral responses. The response distributions of cyclohexane and 1,3-cyclohexadiene did not have significantly different linear slopes at any pair of concentrations tested, even when we attempt to match response size (e.g., comparing the strongest concentration of cyclohexane with the weakest concentration of cyclohexadiene). Thus the effect of two 

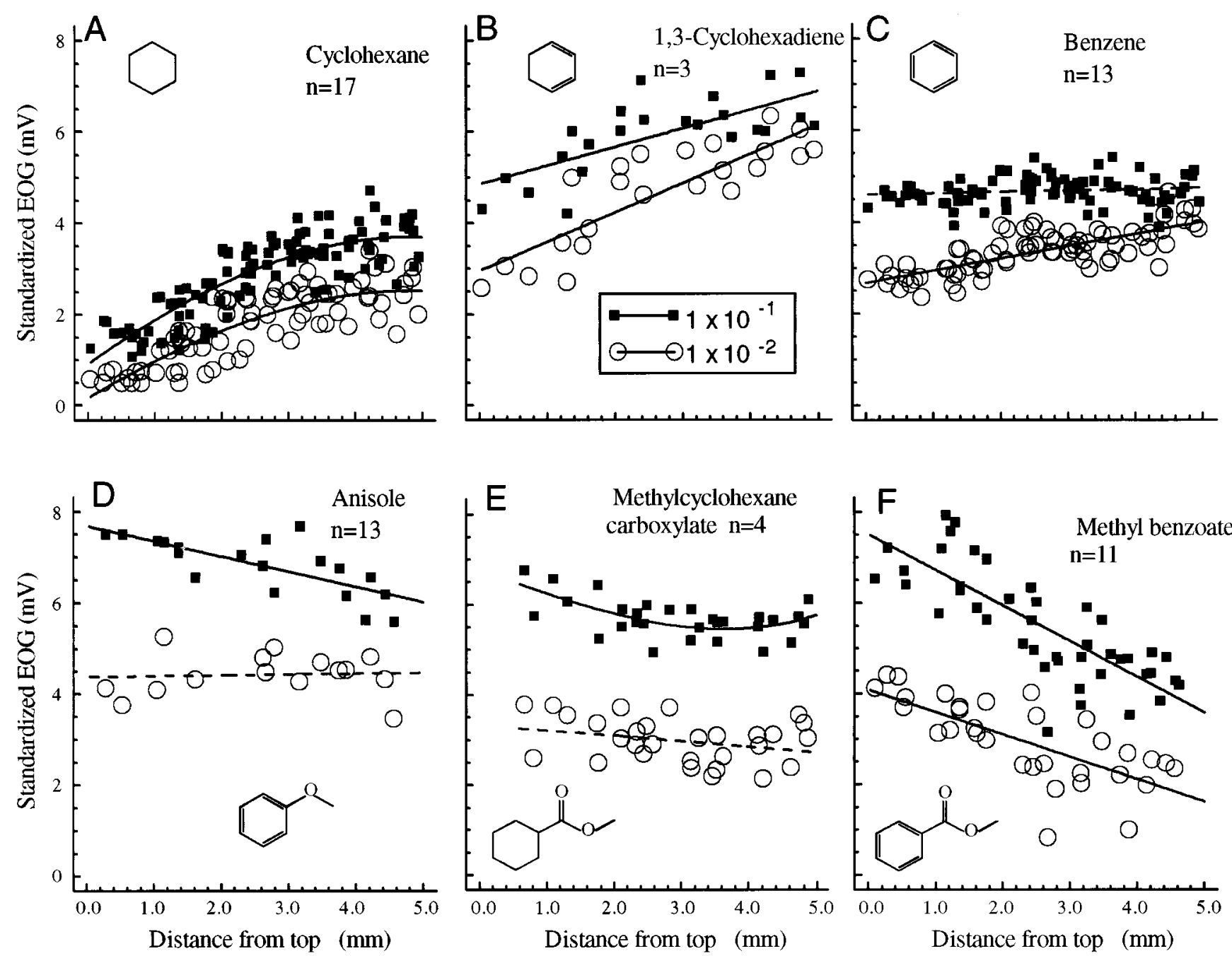

Figure 3. Cyclic odorants. $A$, The response profile for cyclohexane. $B-F$, The effect on the response profile of adding double bonds or oxygen atoms to the ring. In $C-E$, the dashed lines indicate profiles for which the regression was not significant for either a linear or curvilinear function. A dashed line is used merely to indicate the central tendency of the points. It is important to note, however, that even in these cases there is a significant response indicated by a highly significant constant in the regression analysis and that the points are closely grouped around the line, indicating that the line is a reasonable estimate of the response profile.

double bonds was small, as in the straight-chain odorants. In contrast, the response profile slope for benzene was significantly less positive than was the slope for cyclohexane, indicating that the resonance associated with aromatic compounds has a significant effect on the profile.

Adding an ester group $(\mathrm{C}-\mathrm{O}-\mathrm{C}=\mathrm{O})$ to either the cyclohexane or benzene rings, as in methyl cyclohexane carboxylate or methyl benzoate, respectively, produced a large change in the slope of the profile. The slope for the methyl benzoate profile was significantly more negative than a linear slope through the methyl cyclohexane carboxylate profile. This may reflect the summation of the effects of the ester and of the aromatic ring. Comparison of the aromatic ether (anisole) and aromatic ester (methyl benzoate) profiles suggests that esters had more powerful effects than ethers in altering the response profile. The comparison of benzene and anisole slopes was significant when the two were compared at the highest concentration but not when the responses were matched for equal magnitude (e.g., benzene at $1 \times 10^{-1}$ and anisole at $1 \times 10^{-2}$ ).

\section{Terpene odorants}

Terpene odors are of interest because they occur in so many odorous materials. Figure $4 A$ shows the profile for isopropyl cyclohexane, which is very similar in structure to the hydrocarbon backbone of the terpene compounds in the remainder of this figure. The response profile for this odorant was not significantly different in slope from that of the cyclohexane concentrations that evoke responses of similar size. The addition of two double bonds in the $\alpha$ terpinene odorant did not produce a profile significantly different in slope from that of the isopropyl cyclohexane profile. However the profile for D-limonene, in which the double bonds are in different positions, had significantly different slopes from that of both the $\alpha$ terpinene and isopropyl cyclohexane profiles. The ketone group in menthone has more effect on the response than do the double bonds of limonene and $\alpha$ terpinene, so that menthone has a positive slope. The addition of the double bonds and the ketone group in D- and L-carvone produces a significantly more negative slope than does the menthone response, although 

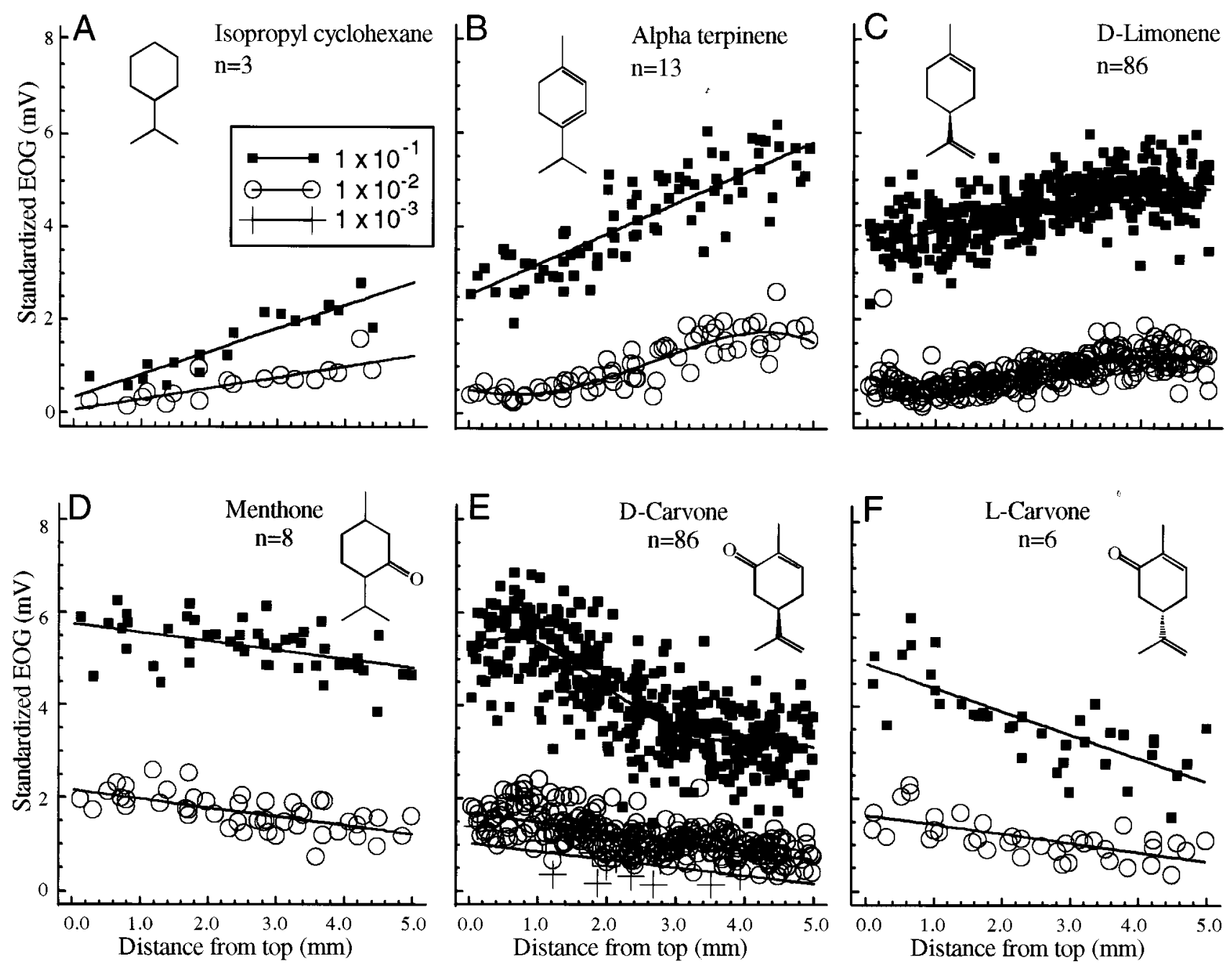

Figure 4. Terpene odorants. $A$, The response to isopropyl cyclohexane, which is very similar in structure to the basic menthane backbone of the terpene compounds illustrated here. $B-F$, The effect on the response profile of adding double bonds and/or ketone groups to the terpene ring. The profiles for the hydrocarbon odorants in $A-C$ all have positive slopes, i.e., successively larger responses on the more ventral electrodes. Note that the enantiomers of carvone in $E$ and $F$ produce nearly identical response profiles.

the profiles for the two carvone forms were not statistically different.

\section{Bicyclic odorants}

As described in our previous publication (Scott and Brierley, 1999), the bicyclic terpene 1,8-cineole produced a strongly nonlinear profile with a peak in the intermediate ventral region, in spite of the presence of an oxygen atom. The third-order equation fit the profiles for this odor significantly better at each concentration from $1 \times 10^{-1}$ to $1 \times 10^{-3}$. The ratio of the coefficients of determination $\left(R^{2}\right.$ indicating the proportion of the variance accounted for by the regression) ranged from 1.25 for the lowest concentration to 1.57 for the highest concentration. Figure 5 tests whether that profile shape was common to all bicyclic compounds or to ethers spanning the cyclohexane ring. The bicyclic hydrocarbon norbornane evoked a response profile not significantly different from that of heptane (Fig. 1) or cyclohexane (Fig. 3), and an ether spanning the 1,4 positions of the ring (Fig. $5 B, C$ ) had very little effect on the response. Of these profiles, only 1,4cineole at $1 \times 10^{-2}$ was significantly nonlinear. Addition of a ketone to other positions on the ring produced a curvilinear response in the case of camphor (Fig. $5 E$ ), although the curvature was small relative to that for 1,8-cineole (Fig. 5D). Norcamphor (Fig. $5 F$ ), with a more exposed ketone, had no significant curvature but had a slight positive slope close to that of menthone (Fig. $4 D)$. For none of the other odorants was the nonlinear effect as great as that for 1,8-cineole. For example, the ratio of the coefficients of determination for camphor was 1.40 at $1 \times 10^{-1}$ but was 1.15 at $1 \times 10^{-2}$.

\section{Comparison of other odorants \\ Linear profiles}

To make an overall comparison of the response profiles of the 40 odors in our sample, we chose to use the linear slope as the major comparison along the $x$-axes of Figure 6 . On the $y$-axes, we represented the proportion of the variance accounted for by a simple linear regression as opposed to a regression model using first-, second-, and third-order terms (see Materials and Methods). On these plots, profiles that were nearly linear, including those for isopropyl cyclohexane and menthone, are found at the top, whereas the 1,8-cineole profile lies close to the $50 \%$ level. 

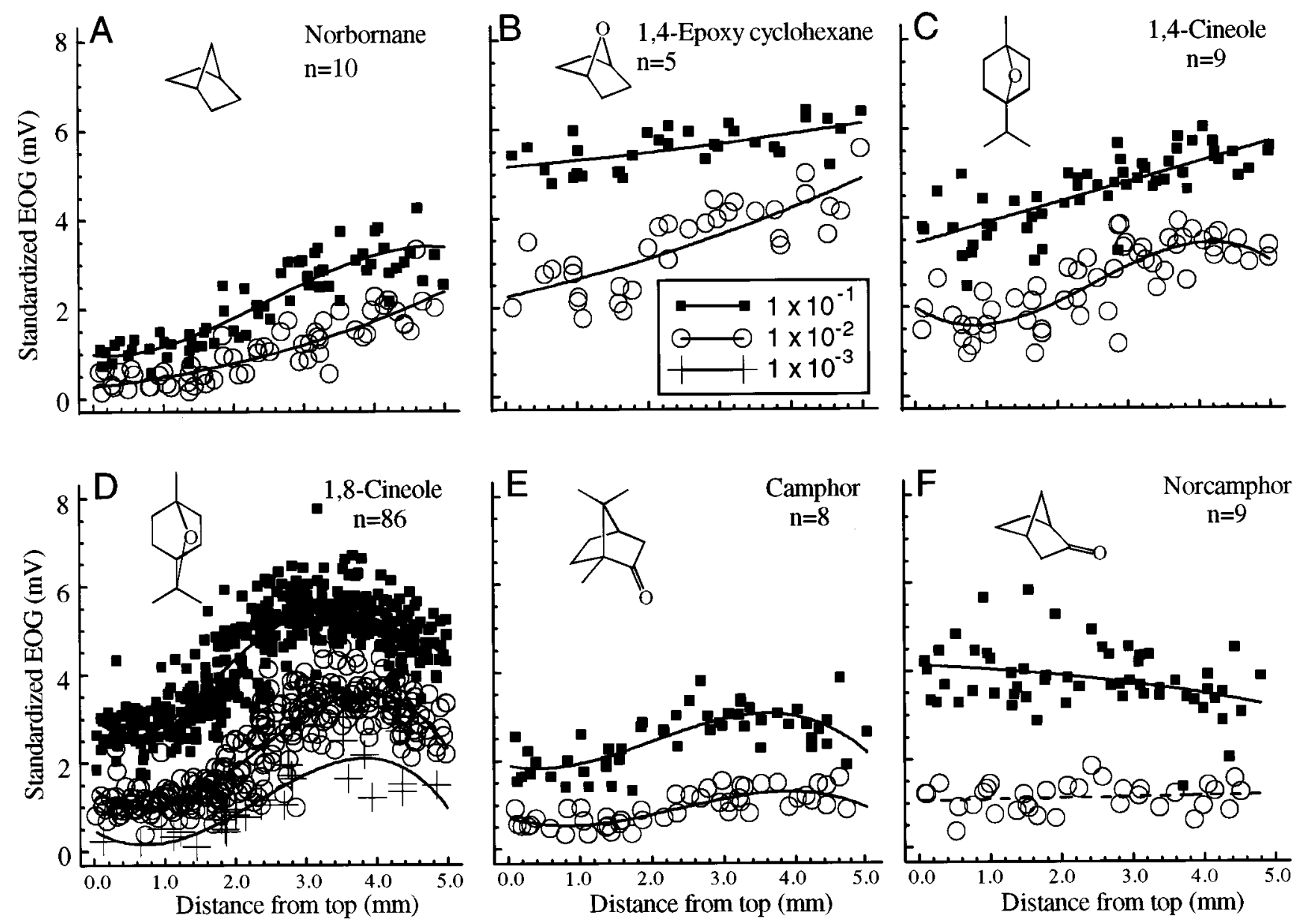

Figure 5. Bicyclic odorants. A, The saturated bicyclic compound norbornane produced responses with a positive slope similar to that of other saturated hydrocarbon odorants. $B-F$, Addition of ether or carbonyl groups altered that slope. For 1,8-cineole and, to a much lesser extent, camphor, there is a marked curvature to the profile with the peak response occurring $\sim 3-4 \mathrm{~mm}$ from the top of endoturbinate IV.

Because most of the profiles were essentially linear, it was appropriate to use the slope for these summary figures.

The odorants were matched as closely as possible for average response magnitude. For example, the average response size for norbornane at $1 \times 10^{-1}$ (Fig. $5 A$ ) was approximately equal to the average response magnitude for methyl benzoate at $1 \times 10^{-2}$ (Fig. $3 F$ ), even though the slopes were opposite. This match was not perfect; for example the heptanoic acid (Fig. 1C) response was a little smaller. The purpose of this matching was to reduce the possibility that the profiles were differentially affected by saturation of the response at high concentrations. There was a suggestion of such an effect in the profiles for 1,3,5-hexatriene (Fig. $1 F$ ), which had a steeper slope at $1 \times 10^{-2}$ than at $1 \times 10^{-1}$. Because there was no concentration tested at which the average response magnitude for this odorant matched those for heptanoic acid, we excluded this odorant from these plots. For the same reason of inability to match response magnitude, we also excluded 1,3-hexadiene and the cyclohexadienes. Of those excluded compounds, 1,3,5-hexatriene and 1,3-hexadiene would have plotted at the far right at the lowest tested concentration. The cyclohexadienes would have plotted further left, near the $\alpha$ terpinene profile.

The characterization of odorant profiles in these plots by a single parameter allowed clearer comparisons than were possible with Figures 1-5. These figures support the conclusion that hydrocarbon odors evoked larger ventral responses (i.e., positive slopes), whereas odorants with carbonyl groups (i.e., an oxygen double bonded to a carbon) produced profiles with negative slopes. This is true among the aliphatic and cyclic odorants (Fig. $6 A$ ), terpene odorants (Fig. 6B), and bicyclic odorants (Fig. 6C). This figure also shows that there were significant differences among the hydrocarbons. These differences were assessed by comparison of the linear slope through the profiles using Equations 4 and 5. The profiles for straight-chain odorants had significantly steeper slopes than did those for cyclic odorants, and the aromatic odorants had even flatter profiles (Fig. 6A). There were also differences among the odorants with carbonyl groups. Interposing carbon or even single-bonded oxygen atoms between the carbonyl group and a ring significantly altered the slope (Fig. 6D). The slopes for most of the terpene ketones (Fig. 6B) were similar to those for benzaldehyde and methyl benzoate (Fig. 6D), but cyclohexane ester profiles (Fig. $6 A$, odorants 15,18 ) had significantly less negative slopes. This suggests that the resonance within the aromatic ring is important.

On the other hand, ethers did not usually have very strong effects on the profile slopes. Although the profile slopes for benzene and anisole were significantly different, the linear profile slopes for 1,4-epoxy cyclohexane, 1,4-cineole, and 1,8-cineole 

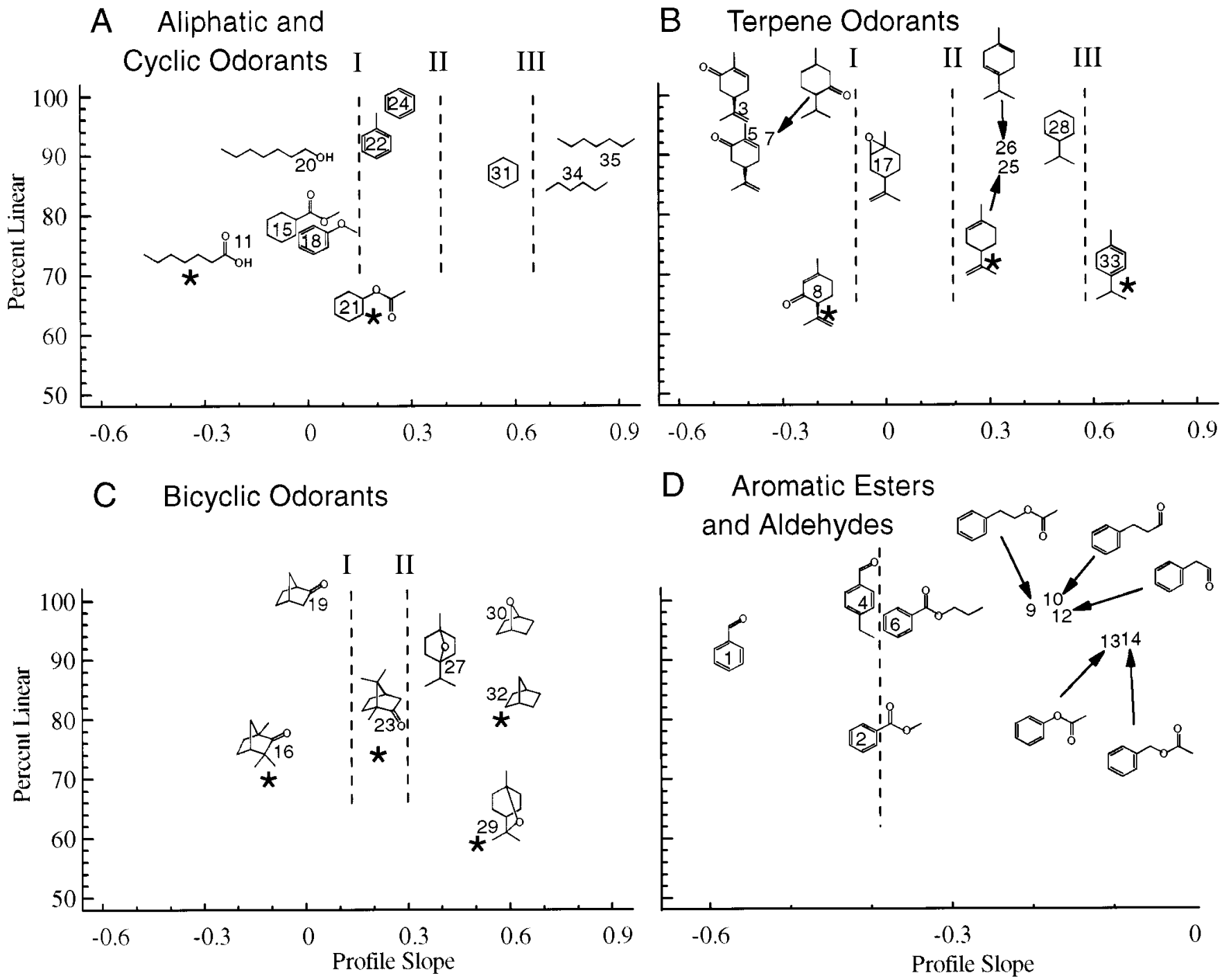

Figure 6. Plots summarizing the response profiles for most of the odorants in this paper. The $x$-axis shows the linear slope for each profile. The $y$-axis shows the ratio of the variance around a linear regression to the variance around a third-order regression. This ratio was close to 1.0 for D-carvone but was only $\sim 0.6$ for 1,8 -cineole. The data are presented by type of compound to avoid crowding. The identification numbers indicating the position of each odorant in this space are in order of the linear slope, with odorant 1 (benzaldehyde) having the greatest negative slope. The asterisks indicate profiles for which there was a significant nonlinear response $(p<0.01)$ by an $F$ test $($ Eq. 3$)$ comparing the linear and nonlinear regression analyses at the concentration illustrated. A, All profiles to the left of the dashed line labeled $I$ were significantly different from all others (with the exception of anisole that was not different from toluene). All profiles to the left of the dashed lines marked $I I$ and $I I I$ were significantly different from those to the right of those lines. B, Similar significant differences were demarcated by the dashed lines in this panel. $C$, The camphor profile was significantly different from all other profiles (except that of norbornane). $D$, The odorants to the left of the dashed line were not significantly different from each other but were significantly different from the hydrocinnamaldehyde, phenylacetaldehyde, and benzyl acetate profiles. At lowered criteria $(p<0.05)$ the methyl benzoate profile was different from the phenyl acetate and phenethyl acetate profiles. Note that the $x$-axis of $D$ corresponds to the left $40 \%$ of that of the other panels. Odorant codes, The numbers of rats tested are indicated for each odorant in parentheses, followed by a lowercase letter showing the concentration code as follows: a, $1 \times 10^{-2}$; b, $3 \times 10^{-2}$; and c, $1 \times 10^{-1}$. 1, Benzaldehyde $(n=9 ; \mathrm{b}) ; 2$, methyl benzoate $(n=11 ; \mathrm{a}) ; 3$, D-carvone $(n=78 ; \mathrm{b}) ; 4$, ethyl benzaldehyde $(n=12 ; \mathrm{b}) ; 5$, L-carvone $(n=6 ; \mathrm{b}) ; 6$, propyl benzoate $(n=8 ; \mathrm{b}) ; 7$, menthone $(n=8 ; \mathrm{b}) ; 8$, isopiperiterone $(n=5 ;$ a); 9 , phenethyl acetate $(n=10$; c); 10 , hydrocinnamaldehyde $(n=10 ; \mathrm{c}) ; 11$, heptanoic acid $(n=11 ; \mathrm{c}) ; 12$, phenylacetaldehyde $(n=5 ; \mathrm{c}) ; 13$, phenyl acetate $(n=9 ; \mathrm{b}) ; 14$, benzyl acetate $(n=12 ; \mathrm{b}) ; 15$, methyl cyclohexane carboxylate $(n=4 ; \mathrm{c}) ; 16$, fenchone $(n=12 ; \mathrm{a}) ; 17$, limonene oxide $(n=5 ; \mathrm{b}) ; 18$, anisole $(n=10 ; \mathrm{b}) ; 19$, norcamphor $(n=9 ; \mathrm{a}) ; 20$, heptanol $(n=10 ; \mathrm{a}) ; 21$, cyclohexyl acetate $(n=4 ; \mathrm{c}) ; 22$, toluene $(n=11 ; \mathrm{a}) ; 23$, camphor $(n=8 ; \mathrm{c}) ; 24$, benzene $(n=13 ; \mathrm{a}) ; 25$, D-limonene $(n=78 ; \mathrm{b}) ; 26$, ( terpinene $(n=7 ; \mathrm{b}) ; 27,1,4$-cineole $(n=9 ; \mathrm{a}) ; 28$, isopropyl cyclohexane $(n=3 ; \mathrm{c}) ; 29,1,8$-cineole $(n=77 ; \mathrm{a}) ; 30,1,4$-epoxy cyclohexane $(n=5 ; \mathrm{a}) ; 31$, cyclohexane $(n=17 ; \mathrm{b}) ; 32$, norbornane $(n=10 ; \mathrm{c}) ; 33, \alpha$ terpinene $(n=13 ; \mathrm{b}) ; 34$, hexane $(n=15 ; \mathrm{b})$; and 35 , heptane $(n=8 ; \mathrm{b})$.

were not significantly different from the slopes for norbornane and cyclohexane. Limonene oxide (Fig. 6B, odorant 17) was the ether with the greatest difference in profile slope from that of the homologous compounds such as limonene or isopropyl cyclohexane. However, as noted above, the profile for limonene oxide was not as steep as that for the ketones.

\section{Nonlinear profiles}

Some of the profiles, notably that of 1,8-cineole and heptanoic acid, had significantly nonlinear response profiles, as shown by their position significantly below the top of Figure 6. Those for which the nonlinearity of the profiles was highly significant $(p<$ 
0.01 by $F$ test) are marked with asterisks in Figure 6. The $y$-axis of the plot is intended solely to illustrate those profiles for which the linear slope was not a good fit and not as a valid descriptor of the profiles. For example, the cyclohexyl acetate profile was linear at other concentrations, whereas the 1,8-cineole profile was strongly nonlinear at all concentrations. For the bicyclic odorants, it appears that the nonlinear profiles were associated with the presence of methyl groups that provide some steric hindrance of the ether or carbonyl groups. Testing this hypothesis will require additional data.

\section{DISCUSSION}

There is a long series of observations [Mustaparta (1971); Kauer and Moulton (1974); for review of other reports, see Ezeh et al. (1995); Scott et al. (1996, 1997)] showing preferential activation of parts of the olfactory epithelium by particular odorants. The present study proposes some consistent relationships that hold across a series of chemical structures. It is premature to try to state a general rule for all of these stimuli, but it is clear that for any of the homologous sets of stimuli (i.e., aliphatic, terpene, cyclic, bicyclic, and aromatic) that the more polar compounds (represented by carbonyl groups compared with hydrocarbons or ethers) are shifted in the direction of having larger dorsal responses. It is also clear that there are important differences between the hydrocarbons of these different odorant sets, especially for comparison of the aromatic and alkane odorants.

These results expand on our previous results by detailing the effects of hydrocarbons and polar compounds across a range of chemical structures and applying those odorants to an opened epithelium where we could observe regions where the responses were quite different. There is an important difference from our previous study of odorant structure (Scott et al., 1996), which was conducted by penetrating the olfactory epithelium from the outside surface, that is, through the lamina propria and nerve layer. That approach had two important consequences: the pattern of odorized airflow was different from that in the present case, and access was restricted to the most medial and most lateral parts of the epithelium. The airflow pattern is significant because of the possibility that some odorants might be more extensively sorbed by the lining of the nasal cavity, thus removing them from the air stream before they reached the receptor surface (Hahn et al., 1994; Kent et al., 1996) (Scott-Johnson, Blakley, and Scott, unpublished observations). Our subsequent reports (Scott et al., 1997; Scott and Brierley, 1999) used direct application of odorant to the exposed mucosal surface of the olfactory epithelium to reduce the influence of this effect. We showed that differential responses could be obtained with very simple airflow patterns and that the direction of airflow did not determine the response pattern in that preparation. However, some of the results reported here may differ from those with the intact nasal cavity (Scott et al., 1997), in that responses to some highly polar odorants were proportionally larger in the ventral part of the exposed epithelium than they were in the lateral part of the intact epithelium in the previous study. Thus the data of the present study may give a more realistic appraisal of the direct response of the epithelium but may underestimate the selectivity in the intact nose.

Our subsequent reports using the opened epithelium (Scott et al., 1997; Scott and Brierley, 1999) explored a much larger surface of the epithelium but concentrated on a small number of terpene odorants. One significant finding with that approach was that 1,8-cineole evoked a more complicated response profile than did the other stimuli. That fact could not be appreciated using the intact nose preparation because of restricted access to the epithelium. That observation with 1,8-cineole has been confirmed here and extended to show that other bicyclic ethers also evoke larger ventral responses than dorsal responses, although their profiles do not exactly match that of 1,8 -cineole. This observation shows that the larger ventral responses are not restricted exclusively to hydrocarbons.

Some of the profiles were significantly nonlinear. These included most notably the 1,8-cineole and carboxylic acid responses, but also the camphor and fenchone (profile not shown) responses. For the bicyclic compounds, there is a suggestion that the steric hindrance of the ether or carbonyl group by methyl groups might be an important factor in whether these profiles showed an increased response in the intermediate region of endoturbinate IV. The case of the carboxylic acids was different. For those stimuli, the pattern was nearly opposite, with a slight decreased response in the intermediate region compared with either the dorsal or ventral regions. Although it is possible to imagine a single process that would determine the major slope of most of the profiles shown here, the nonlinear profiles suggest that there are several processes involved in some cases.

The issue of the mechanism(s) producing these profiles is not addressed by these data. We have suggested that they may result from the olfactory gene expression zones as described by Vassar et al. (1994) and Ressler et al. (1993). In that case, there would be a preponderance of particular types of receptors in each different zone. Interestingly, the nearly linear profiles seen here and even in data from individual animals (cf. Scott and Brierley, 1999) do not suggest discrete regions of sensitivity as we might expect from the strictest interpretation of the zonal hypothesis. Nevertheless, these recordings may not have the resolution to test whether the boundaries of the gene expression zones are functional boundaries. Although direct odorant application greatly reduces the chance of differential odorant sorption along the pathway to the receptor sheet, it does not remove the possibility of differential rates of entry of stimuli into the tissue. We have discussed previously the possibility of regional differences in sorption (Scott and Brierley, 1999). We have not seen differences in the kinetics of the responses that would support this interpretation, but this has not been formally studied.

If the general pattern of response seen across endoturbinate IV is general on the olfactory epithelium, then there would be a very definite pattern of input into the olfactory bulb related to a set of odorant chemistry because of the pattern of projection of axons of olfactory sensory neurons (Astic et al., 1987; Schoenfeld et al., 1994). Such a pattern would be consistent with physiology indicating a larger response to carboxylic acid stimuli in the dorsal part of the olfactory bulb (Mori and Yoshihara, 1995; Johnson et al., 1999) and a larger alkane response in the ventral part of the bulb (Mori and Yoshihara, 1995). If our data are borne out on other surfaces of the epithelium, it would suggest that there is a very general pattern of these inputs that extends far beyond the aliphatic odorants considered in those reports. Such a relationship, taken together with recent evidence of a rostral-to-caudal response gradient for the length of acid and aldehyde odorants in the olfactory bulb, suggests a map of chemical properties on the olfactory 
bulb (Johnson et al., 1999; Rubin and Katz, 1999). So far, we have seen no evidence of a regional variation of response to the chain length of aliphatic aldehydes or alkanes in our preparation, and those gradients in the bulb response probably arise from the pattern of selective projection of axons related to particular receptor genes to specific glomeruli (Ressler et al., 1994; Vassar et al., 1994; Mombaerts et al., 1996). One of those receptor types has been shown to be associated with high-chain length specificity among the aliphatic aldehydes (Krautwurst et al., 1998; Zhao et al., 1998). Such high specificity has not yet been demonstrated in olfactory sensory neurons (DuchampViret et al., 1999; Malnic et al., 1999).

The pattern of response demonstrated here would certainly not account for all the variance in the response to odors. For example, we know that in some cases enantiomers of the terpene odorants smell different (Laska and Teubner, 1999) and seem to bind different receptors (Krautwurst et al., 1998); yet at least in the case of carvone, these enantiomers have indistinguishable profiles. Thus the pattern of the responses in the different regions of the epithelium is certainly refined by the pattern of projection from olfactory sensory neurons expressing particular receptor genes onto specific glomeruli. Yet the EOG patterns are probably in register with the projection patterns from the mucosa to the bulb (Astic et al., 1987; Schoenfeld et al., 1994) and thus determine a significant portion of the spatial pattern of the bulbar response to odor. Such observations make it clear that there is a strong spatial pattern in this input. On the other hand, the fact that responses to all odorants can be found in all parts of the epithelium may help us to understand why removing large parts of the olfactory bulb does not remove the response to particular odors, as demonstrated by Slotnick and colleagues (Slotnick et al., 1997; Lu and Slotnick, 1998). Laurent (1999) has written recently a provocative challenge to the idea that the pattern of spatial representation of odorants can have a significant effect in odor coding. Observations such as ours coupled with the demonstration of a spatial localization in the acid or aldehyde response measured with 2-deoxyglucose (Johnson et al., 1999), optical imaging (Rubin and Katz, 1999), or electrophysiology (Yokoi et al., 1995) provide strong evidence of such spatial representation. The spatial localization of response within the olfactory bulb is probably produced by several factors: (1) the regional distribution of sensitivity described here, (2) the effects of sorption along the airway that probably acts to accentuate the separation of polar and nonpolar odorants (Hahn et al., 1994; Ezeh et al., 1995; Kent et al., 1996) (Scott-Johnson, Blakley, and Scott, unpublished observations), (3) the convergence pattern of olfactory receptor neuron axons expressing the same receptor onto small populations of glomeruli (Ressler et al., 1994; Vassar et al., 1994; Mombaerts et al., 1996), and (4) interneuronal interactions within the olfactory bulb (Yokoi et al., 1995). Therefore, it is likely that discrepancies will be evident when different levels of the system are compared. It is too early to state the degree to which any or all of these spatial constraints are essential for odor discrimination.

\section{REFERENCES}

Astic L, Saucier D, Holley A (1987) Topographic relationships between olfactory receptor cells and glomerular foci in the rat olfactory bulb. Brain Res 424:144-152.
Buck L, Axel R (1991) A novel multigene family may encode odorant receptors: a molecular basis for odor recognition. Cell 65:175-187.

Buonviso N, Chaput MA (1990) Response similarity to odors in olfactory bulb output cells presumed to be connected to the same glomerulus: electrophysiological study using simultaneous single-unit recordings. J Neurophysiol 63:447-454.

Duchamp-Viret P, Chaput MA, Duchamp A (1999) Odor response properties of rat olfactory receptor neurons. Science 284:2171-2174.

Ezeh PI, Scott JW, Davis LM (1995) Regional distribution of rat electroolfactogram. J Neurophysiol 73:2207-2220.

Guthrie KM, Anderson AJ, Leon M, Gall C (1993) Odor-induced increases in $c$-fos mRNA expression reveal an anatomical "unit" for odor processing in olfactory bulb. Proc Natl Acad Sci USA 90:3329-3333.

Hahn I, Scherer PW, Mozell MM (1994) A mass transport model of olfaction. J Theor Biol 167:115-128.

Johnson BA, Woo CC, Leon M (1998) Spatial coding of odorant features in the glomerular layer of the rat olfactory bulb. J Comp Neurol 393:457-471.

Johnson BA, Woo CC, Hingco EE, Pham KL, Leon M (1999) Multidimensional chemotopic responses to n-aliphatic acid odorants in the rat olfactory bulb. J Comp Neurol 409:529-548.

Kauer JS, Moulton DG (1974) Responses of olfactory bulb neurons to odor stimulation of small nasal areas in the salamander. J Physiol (Lond) 243:717-737.

Kauer JS, Shepherd GM (1975) Olfactory stimulation with controlled and monitored step pulses of odor. Brain Res 85:108-113.

Kent PF, Mozell MM, Murphy SJ, Hornung DE (1996) The interaction of imposed and inherent olfactory mucosal activity patterns and their composite representation in a mammalian species using voltagesensitive dyes. J Neurosci 16:345-353.

Kleinbaum DG, Kupper LL (1978) Applied regression analysis and other multivariable methods. North Scituate, MA: Duxbury.

Krautwurst D, Yau K-W, Reed RR (1998) Identification of ligands for olfactory receptors by functional expression of a receptor library. Cell 95:917-926.

Kubick S, Strotmann J, Andreini I, Breer H (1997) Subfamily of olfactory receptors characterized by unique structural features and expression patterns. J Neurochem 69:465-475.

Laska M, Teubner P (1999) Olfactory discrimination ability of human subjects for ten pairs of enantiomers. Chem Senses 24:161-170.

Laurent G (1999) A systems perspective on early olfactory coding. Science 286:723-728.

Linster C, Hasselmo E (1999) Behavioral responses to aliphatic aldehydes can be predicted from known electrophysiological responses of mitral cells in the olfactory bulb. Physiol Behav 66:497-502.

Lu XC, Slotnick BM (1998) Olfaction in rats with extensive lesions of the olfactory bulbs: implications for odor coding. Neuroscience 84:849866.

Mackay-Sim A, Kesteven S (1994) Topographic patterns of responsiveness to odorants in the rat olfactory epithelium. J Neurophysiol 71:150-160.

Malnic B, Hirono J, Sato T, Buck LB (1999) Combinatorial receptor codes for odors. Cell 96:713-723.

Mombaerts P, Wang F, Dulac C, Chao SK, Nemes A, Mendelsohn M, Edmondson J, Axel R (1996) Visualizing an olfactory sensory map. Cell 87:675-686.

Mori K, Yoshihara Y (1995) Molecular recognition and olfactory processing in the mammalian olfactory system. Prog Neurobiol 45:585-619.

Mustaparta H (1971) Spatial distribution of receptor-responses to stimulation with different odours. Acta Physiol Scand 82:154-166.

Nef P, Hermans-Borgmeyer I, Artieres-Pin H, Beasley L, Dionne VE, Heinemann SF (1992) Spatial pattern of receptor expression in the olfactory epithelium. Proc Natl Acad Sci USA 89:8948-8952.

Ressler KJ, Sullivan SL, Buck LB (1993) A zonal organization of odorant receptor gene expression in the olfactory epithelium. Cell 73:597-609.

Ressler KJ, Sullivan SL, Buck LB (1994) Information coding in the olfactory system: evidence for a stereotyped and highly organized epitope map in the olfactory bulb. Cell 79:1245-1255.

Rubin BD, Katz LC (1999) Optical imaging of odorant representations in the mammalian olfactory bulb. Neuron 23:499-511.

Schoenfeld TA, Clancy AN, Forbes WB, Macrides F (1994) The spatial organization of the peripheral olfactory system of the hamster. Part I: Receptor neuron projections to the main olfactory bulb. Brain Res Bull 43:183-210. 
Scott JW, Brierley T (1999) A functional map in rat olfactory epithelium. Chem Senses 24:679-690.

Scott JW, Davis LM, Shannon D, Kaplan C (1996) Relation of chemical structure to spatial distribution of sensory responses in rat olfactory epithelium. J Neurophysiol 75:2036-2049.

Scott JW, Shannon DE, Charpentier J, Davis LM, Kaplan C (1997) Spatially organized response zones in rat olfactory epithelium. J Neurophysiol 77:1950-1962.

Slotnick BM, Bell GA, Panhuber H, Laing DG (1997) Detection and discrimination of propionic acid after removal of its 2-DG identified major focus in the olfactory bulb: a psychophysical analysis. Brain Res 762:89-96.

Strotmann J, Wanner I, Helfrich T, Beck A, Meinken C, Kubick S, Breer H (1994) Olfactory neurones expressing distinct odorant receptor sub- types are spatially segregated in the nasal neuroepithelium. Cell Tissue Res 276:429-438.

Vassar R, Ngai J, Axel R (1993) Spatial segregation of odorant receptor expression in the mammalian olfactory epithelium. Cell 74:309-318.

Vassar R, Chao SK, Sitcheran R, Nuñez JM, Vosshall LB, Axel R (1994) Topographic organization of sensory projections to the olfactory bulb. Cell 79:981-991.

Yokoi M, Mori K, Nakanishi S (1995) Refinement of odor molecule tuning by dendrodendritic synaptic inhibition in the olfactory bulb. Proc Natl Acad Sci USA 92:3371-3375.

Zhao H, Ivic L, Otaki JM, Hashimoto M, Mikoshiba K, Firestein S (1998) Functional expression of a mammalian odorant receptor. Science 270:237-242. 